\title{
Randomised placebo-controlled safety and tolerability trial of FK506 (tacrolimus) for pulmonary arterial hypertension
}

\author{
Edda Spiekerkoetter ${ }^{1,2}$, Yon K. Sung ${ }^{1,2}$, Deepti Sudheendra ${ }^{1,2}$, Valerie Scott ${ }^{1,2}$, \\ Patricia Del Rosario ${ }^{1,2}$, Matthew Bill ${ }^{1,2}$, Francois Haddad ${ }^{3}$, Janel Long-Boyle ${ }^{4}$, \\ Haley Hedlin ${ }^{5}$ and Roham T. Zamanian ${ }^{1,2}$
}

Affiliations: 'Division of Pulmonary and Critical Care Medicine, Dept of Medicine, Stanford University, Stanford, CA, USA. ${ }^{2}$ Vera M. Wall Center for Pulmonary Vascular Disease, Dept of Medicine, Stanford University, Stanford, CA, USA. ${ }^{3}$ Division of Cardiology, Dept of Medicine, Stanford University, Stanford, CA, USA. ${ }^{4}$ Dept of Clinical Pharmacy, University of California, San Francisco, CA, USA. ${ }^{5}$ Division of Biomedical Informatics Research, Dept of Medicine, Stanford University, Stanford, CA, USA.

Correspondence: Edda Spiekerkoetter, Division of Pulmonary and Critical Care Medicine, Stanford University Medical Center, Room H3143, 300 Pasteur Drive, Stanford, CA 94305, USA. E-mail: eddasdastanford.edu

@ERSpublications

Low-level FK506 (tacrolimus) is well tolerated in stable PAH and increases BMPR2 in subsets of patients http://ow.ly/t5c530cE1vx

Cite this article as: Spiekerkoetter E, Sung YK, Sudheendra D, et al. Randomised placebo-controlled safety and tolerability trial of FK506 (tacrolimus) for pulmonary arterial hypertension. Eur Respir J 2017; 50: 1602449 [https://doi.org/10.1183/13993003.02449-2016].

ABSTRACT Pulmonary arterial hypertension (PAH) is a devastating disease characterised by occlusive pulmonary vasculopathy. Activation of bone morphogenetic protein receptor 2 (BMPR2) signalling by FK506 (tacrolimus) reverses occlusive vasculopathy in rodent PAH models. Here, we determined the safety and tolerability of low-level FK506 therapy in stable PAH patients.

We performed a randomised, double-blind, placebo-controlled, 16-week, single-centre, phase IIa trial in PAH patients with New York Heart Association functional class II/III symptoms using three FK506 target levels $\left(<2,2-3\right.$ and 3-5 ng.mL $\left.{ }^{-1}\right)$. 23 patients were randomised and 20 patients completed the trial.

FK506 was generally well tolerated, with nausea/diarrhoea being the most commonly reported adverse event and no observation of line infections in patients on intravenous prostacyclin therapy. PAH patients had significantly lower BMPR2 expression in peripheral blood mononuclear cells versus healthy controls $(n=13 ; p=0.005)$, which improved after FK506 treatment. While we observed that some patients responded with a pronounced increase in BMPR2 expression as well as improvement in 6-min walk distance, and serological and echocardiographic parameters of heart failure, these changes were not significant.

Low-level FK506 is well tolerated and increases BMPR2 in subsets of PAH patients. These results support the study of FK506 in a phase IIb efficacy trial.

This article has supplementary material available from erj.ersjournals.com

Received: Dec 142016 | Accepted after revision: June 072017

This study is registered at ClinicalTrials.gov with identifier number NCT01647945.

Support statement: Wall Center for Pulmonary Vascular Disease and SPARK, both at Stanford University and NIH CTSA award UL1 RR025744 (Principal Investigator: Harry Greenberg). Funding information for this article has been deposited with the Crossref Funder Registry.

Conflict of interest: Disclosures can be found alongside this article at erj.ersjournals.com

Copyright @ERS 2017 


\section{Introduction}

Although significant advances have been made in the treatment of pulmonary arterial hypertension $(\mathrm{PAH})$, the disease is still characterised by significant morbidity and mortality due to progressive pulmonary obstructive vasculopathy despite effective pulmonary vasodilation [1]. Targeting the bone morphogenetic protein receptor 2 (BMPR2) pathway has emerged as a novel treatment strategy for reversing obstructive vasculopathy in experimental pulmonary hypertension $[2,3]$. Genetic, histological and biomarker studies demonstrate that the BMPR2 pathway is downregulated in $\mathrm{PAH}$, and suggest that the BMPR2 pathway could be targeted therapeutically [4-8]. BMPR2 gene therapy attenuates experimental hypoxic pulmonary hypertension in rats and selective enhancement of BMPR2 by the ligand BMP9 reverses experimental pulmonary hypertension $[3,9,10]$. In a high-throughput screen of more than 3600 US Food and Drug Administration-approved drugs, we previously identified FK506 (tacrolimus) as a potent BMPR2 activator that reversed endothelial dysfunction in PAH patient cells and experimental pulmonary hypertension [2]. Compassionate use of low-dose FK506 (trough level 1.5-2.5 ng.mL ${ }^{-1}$ ) in three patients with end-stage PAH for 12 months improved heart failure symptoms, New York Heart Association (NYHA) functional class, 6-min walk distance (6MWD) and N-terminal pro-brain natriuretic peptide (NT-proBNP), and increased BMPR2 expression in peripheral blood mononuclear cells (PBMCs) [8].

Whereas the short- and long-term toxicity of FK506 at immunosuppressive doses after transplantation is well known (target trough levels 5-15 ng. $\mathrm{mL}^{-1}$ ), the side-effect profile of a lower target blood level of FK506 (2-5 ng. $\left.\mathrm{mL}^{-1}\right)$ in PAH patients with different comorbidities and concomitant medications has not been studied. This phase IIa trial examined the safety of a 16-week treatment with low-level FK506 in $\mathrm{PAH}$ patients. In addition, we sought to determine the impact of therapy on clinical parameters, including symptoms, exercise tolerance, right ventricular function by echocardiography, serological heart failure markers as well as BMPR2 signalling in PBMCs.

\section{Material and methods}

\section{Study design}

The tacrolimus in PAH (TransformPAH) trial (ClinicalTrials.gov: NCT01647945) was a randomised, double-blind, placebo-controlled, 16-week, single-centre study. Participants were randomised into four study arms: placebo, low-level FK506 (<2 ng.mL $\left.{ }^{-1}\right)$, medium-level FK506 (2-3 ng.mL $\left.{ }^{-1}\right)$ and high-level FK506 (3-5 ng.mL $\left.{ }^{-1}\right)$. Of note, even the high-level FK506 arm in our study had levels that are normally not targeted for immunosuppression as they only convey a mild immunosuppressive effect. The planned study size was 40 patients with 10 patients per treatment group, although no formal power calculation was performed. The trial was approved by the Institutional Review Board at Stanford University (Stanford, CA, USA). All participants provided written informed consent. The research was conducted according to the principles of the Declaration of Helsinki.

\section{Patient population}

All patients followed in the Stanford Adult Pulmonary Hypertension Clinic (Stanford University Medical Center, Stanford, CA, USA) with the diagnosis of PAH were screened in a prospective manner. We included subjects age $\geqslant 18$ and $<70$ years, NYHA functional class I-IV on maximal therapy, PAH documented by right heart catheterisation (pulmonary arterial pressure $\geqslant 25 \mathrm{mmHg}$, pulmonary artery wedge pressure $<15 \mathrm{mmHg}$ and pulmonary vascular resistance $>240 \mathrm{dyn} \cdot \mathrm{s} \cdot \mathrm{cm}^{-5}$ ) that was idiopathic (I), heritable $(\mathrm{H})$ or associated $(\mathrm{A}) \mathrm{PAH}$, including connective tissue disease (CTD), drugs and toxins $(\mathrm{D}+\mathrm{T})$, corrected congenital heart disease (CHD) and porto-pulmonary hypertension. Subjects had to be clinically stable on active $\mathrm{PAH}$ treatment for $\geqslant 3$ months.

Excluded were subjects with group 2-5 pulmonary hypertension, restrictive or obstructive lung disease, significant left heart disease based on screening echocardiography, chronic kidney disease, or moderate to severe hepatic dysfunction (see supplementary material for detailed inclusion and exclusion criteria). Subjects using bosentan were excluded because animal studies had shown that FK506 might increase bosentan levels (per drug label information of bosentan; www.drugs.com).

\section{Study procedures}

The medical staff and study coordinators identified potentially eligible subjects. The recruitment goal was to randomise 10 subjects per arm. Using block randomisation, subjects were assigned in a 1:1:1:1 manner by a web-based computerised system to either placebo or one of three FK506 treatment arms. We utilised 0.5 and $1.0 \mathrm{mg}$ FK506 pills and matching placebo (produced by the University of California San Francisco Compounding Support Pharmacy, San Francisco, CA, USA; see supplementary material). All patients were started at $1.5 \mathrm{mg}$ by mouth once daily with dose adjustment according to a pre-specified titration algorithm (supplementary figure E1). Tacrolimus levels reported below the level of quantification 
$\left(<2 \mathrm{ng} \cdot \mathrm{mL}^{-1}\right)$ were entered into the dosing algorithm at $1 \mathrm{ng} \cdot \mathrm{mL}^{-1}$. Subjects started on day 0 , had their blood drawn on day 4 and, depending on the level, received instructions on dose modification if needed. During drug titration, subjects were required to have blood levels drawn every 4 days; subjects at desired goal FK506 levels required drug level checks every 2 weeks. The placebo arm was titrated using a sham titration algorithm developed a priori (see supplementary material). All subjects and study personnel were masked to treatment assignment until study completion. An unblinded physician (Y.K.S.) and a research pharmacist monitored and titrated FK506 doses without being involved in patient assessment. Subjects were evaluated at baseline, 4, 8 and 16 weeks. At each study visit, blood for safety monitoring and NT-proBNP was drawn, and a 6-min walk test was performed. The trial medication was stopped at 16 weeks and subjects presented for an 18-week visit to capture any potential adverse outcomes after study drug cessation. Telephone contact was made by study nurse coordinators during weeks 2, 6, 10 and 12 to assess symptoms and adverse events (supplementary figure E2).

\section{Study monitoring}

Details of the study monitoring are provided in the supplementary material.

\section{Outcome measures}

The primary objective of our trial was to demonstrate the safety and tolerability of FK506, and to show the feasibility of targeting different low-dose FK506 ranges. Safety and tolerability were assessed by adverse events, vital signs, serum creatinine, haemoglobin and white blood cell (WBC) count.

Secondary objectives included changes in 6MWD, NYHA functional class, NT-proBNP, diffusion capacity of the lung for carbon monoxide (DLCO), echocardiographic measures of right ventricular function, including right ventricular fractional area change (RV-FAC), tricuspid annular plane systolic excursion (TAPSE), right ventricular global longitudinal strain (RV-GLS) and time to clinical worsening (TTCW). TTCW was defined as a combined end-point including the need for uptitration or addition of PAH therapies, worsening NYHA functional class, hospitalisation for right heart failure, lung transplantation, atrial septostomy and all-cause mortality.

As exploratory objectives, we examined the change in PBMCs and plasma biomarkers that would verify that the BMPR2 pathway was targeted by the treatment. Specifically, the following biomarkers were measured by quantitative reverse transcriptase PCR (Applied Biosystems, Foster City, CA, USA): BMPR2, inhibitor of differentiation 1 (Id1), Cofilin-1, Lim domain kinase 1 (LIMK-1), Smad ubiquitination regulatory factor 1 (SMURF-1), and microRNAs miR21 and miR27a. Plasma interleukin (IL)-6 levels were assayed by the Quantikine human IL-6 ELISA (R\&D Systems, Minneapolis, MN, USA). PBMCs from 13 healthy controls were used for comparison. Lastly, we sought to identify potential "FK506 responders" with regard to their change in BMPR2 expression as well as their clinical response (an approach to guide patient enrichment strategies for a potential phase IIb/III efficacy trial).

\section{Statistical analysis}

Baseline patient characteristics are presented as median, interquartile range (IQR), count and percentage. Drug levels and clinical/serological biomarkers were displayed graphically. For clinical and serological biomarkers (primary, secondary and exploratory end-points), we compared the change between the baseline and 16-week measures using a linear regression with a random intercept for subjects to account for within-subject correlation. The biomarker levels were compared across 1) study arms (placebo, low-, medium- and high-level FK506) and 2) study drug (all FK506 levels) versus placebo. The data were analysed with an intent-to-treat principle when possible (see supplementary material for detailed statistical analysis). For the exploratory biomarkers BMPR2 and Id1, the baseline and 16-week values were compared with healthy controls using the Mann-Whitney-Wilcoxon test. As a post hoc analysis, we also compared the changes in BMPR2 and Id1 in subjects who had a more pronounced clinical response than others, defined as: increase in $6 \mathrm{MWD}$ of $>40 \mathrm{~m}$, decrease in NT-proBNP of $\geqslant 200 \mathrm{pg} \cdot \mathrm{mL}^{-1}$, increase in RV-FAC of $\geqslant 1.8 \%$ or decrease in RV-GLS of $\geqslant 2.5 \%$ (using the Mann-Whitney-Wilcoxon test) [11]. All analyses were performed using R version 3.2 (www.r-project.org). Statistical significance was specified $a$ priori at a p-value of 0.05 . All formal tests were intended to generate hypotheses for future studies.

\section{Results}

\section{Study conduct}

During the 19-month study period, 459 subjects were screened (figure 1), 51 subjects were approached for consent and 23 subjects agreed to participate in the trial. Each study arm included five or six subjects. The first patient was randomised in September 2012 and the last patient last visit occurred in April 2014. Although a recruitment frequency of one to two patients per month was maintained, the study was ultimately stopped short of the planned sample size of 40 by the investigators due to limited study subjects 


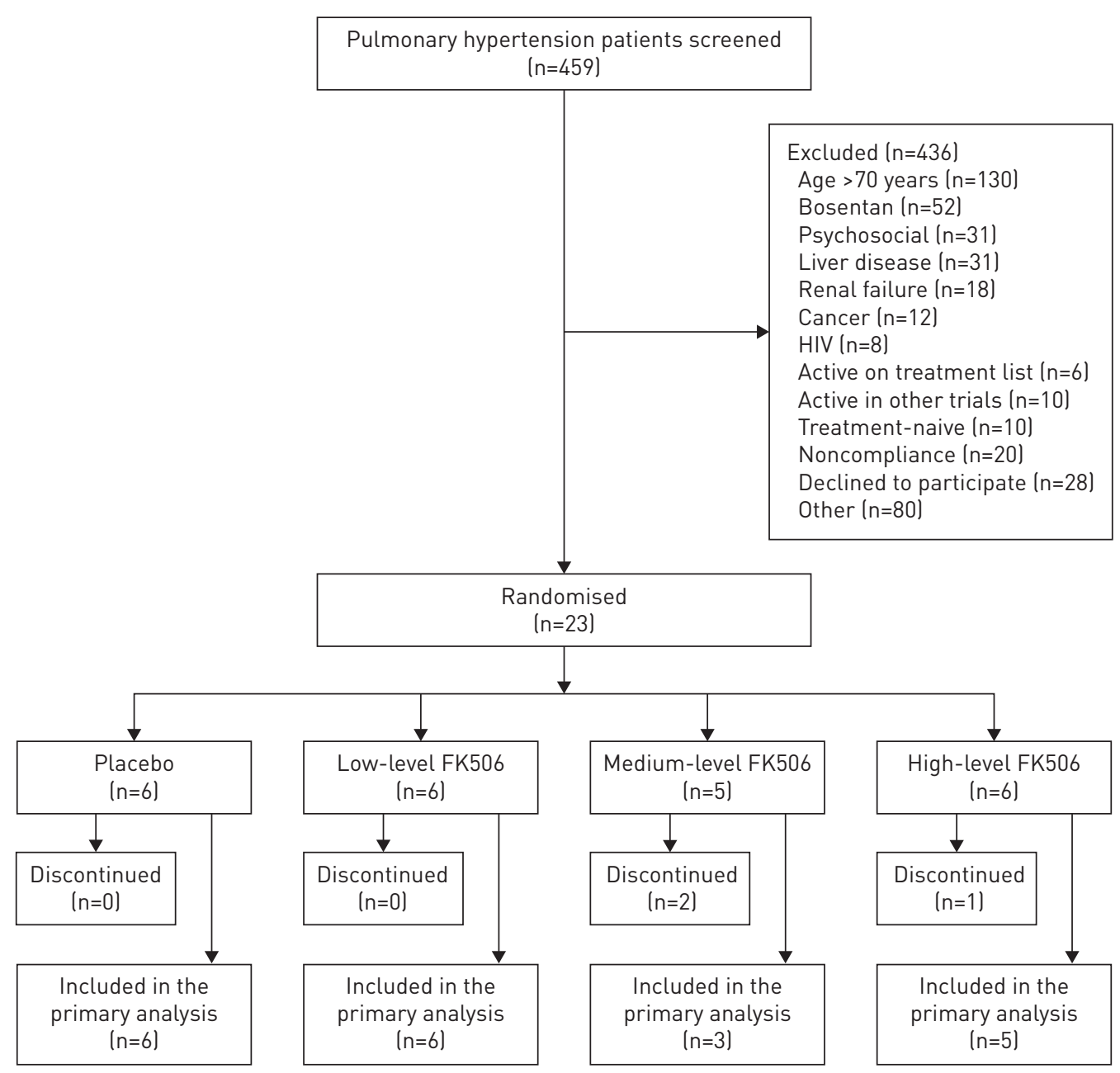

FIGURE 1 Screening, randomisation and follow-up.

after 2 years of active screening, highlighting the difficulty of completing a substantial PAH study at a single centre. The most common reasons for trial exclusion were age $>70$ years and concomitant use of bosentan. Of the 23 enrolled participants, 20 subjects completed the 18 -week assessment at study end and three patients discontinued the study.

\section{Baseline demographics and patient characteristics}

The entire study population had a median (IQR) age of 41 (34-52) years and was mostly female (table 1). In terms of aetiology of PAH, patients were diagnosed with IPAH ( $\mathrm{n}=6), \mathrm{HPAH}$ with known BMPR2 mutation $(n=4)$ (equally randomised to the four arms), D+T-APAH $(n=5)$, CTD-APAH $(n=5)$ and CHD-APAH $(n=3)$. Most patients had either mild or well-treated PAH as evident by predominantly NYHA functional class II (78\%), high median (IQR) baseline 6MWD (526 (472-567) $\mathrm{m}$ ) and low median (IQR) NT-proBNP (98 (57-328) pg.mL ${ }^{-1}$ ). Approximately 50\% of the patients were on triple therapy, $25 \%$ on dual therapy and $25 \%$ on monotherapy. Subjects were evenly distributed across study arms with regard to aetiology, disease severity and concomitant medication.

\section{Feasibility of targeting low-dose FK506}

Following our FK506 dosing protocol, we found that on average seven blood draws were required to discriminate between the median trough levels in the placebo, low-, medium- and high-level FK506 arms (figure 2a). This time-point corresponded to $\sim 50$ days after initiation of FK506 (figure 2b), longer than the estimated 4 weeks we anticipated to reach stable levels. Despite the reported broad range of interperson bioavailability of oral FK506 at immunosuppressive doses of 5-65\% [12, 13] as well as the abundant drugdrug interactions with concomitant drugs metabolised by the cytochrome P450 system (specifically CYP3A4), we observed a clear dose response with a relatively small IQR (figure 2 c; no statistical analyses 

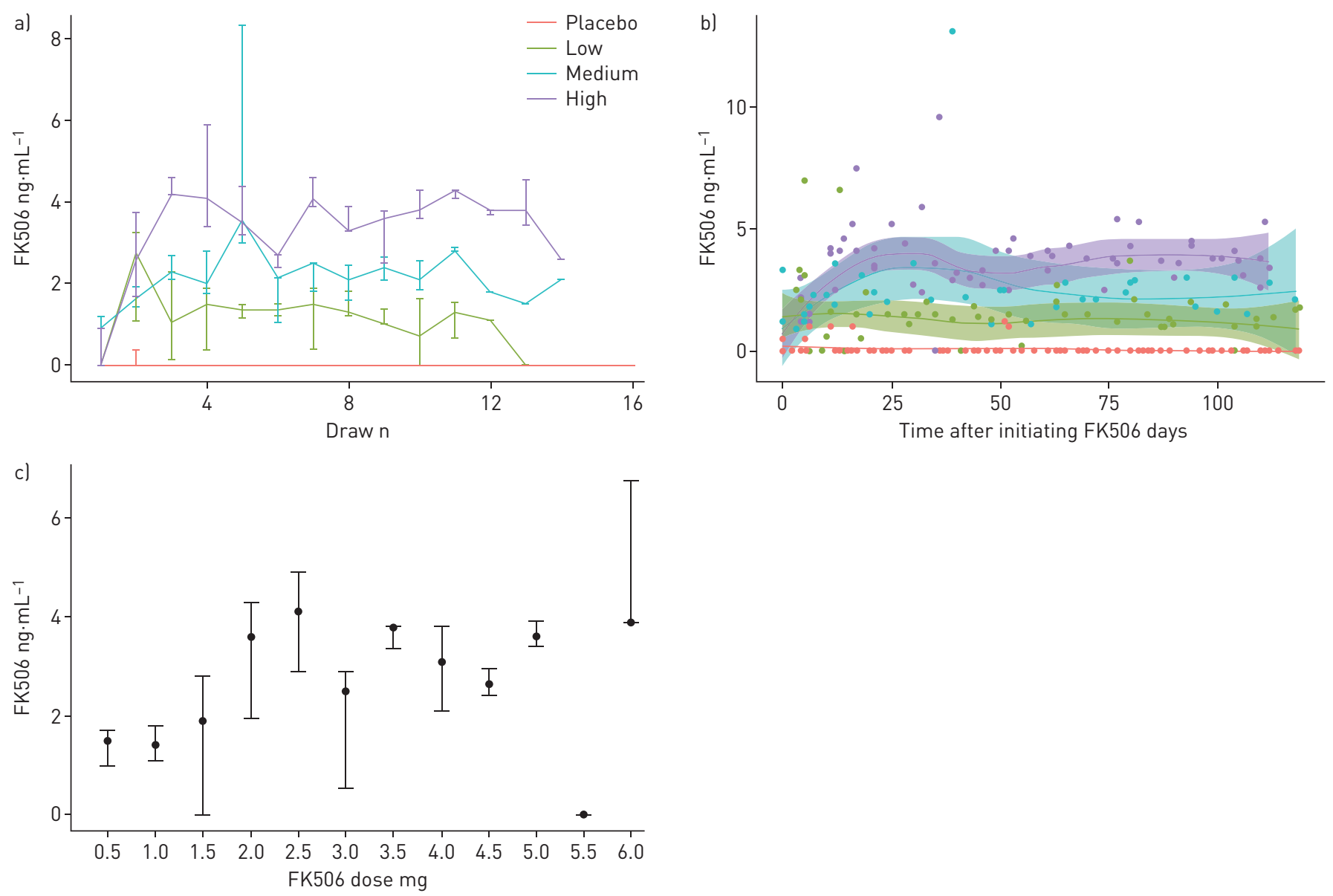

FIGURE 2 Feasibility of achieving distinct FK506 blood levels. FK506 drug levels in the assigned treatment group (placebo, low ( $<2 \mathrm{ng} \cdot \mathrm{mL}^{-1}$ ), medium (2-3 ng. $\left.\mathrm{mL}^{-1}\right)$ and high (3-5 $\left.\mathrm{ng} \cdot \mathrm{mL}^{-1}\right)$ ) are represented as a function of a) the number of blood draws and b) days after initiation of study. c) Correlation between drug level (ng $\mathrm{mL}^{-1}$ ) and FK506 dose (mg) in all treatment groups. FK506 doses are reflective of at least three patients, presented as median with interquartile range and single observations as a single bar.

were done for this figure, it is solely for descriptive purposes). Our results suggest a favourable and narrow range of bioavailability with low-level FK506 in PAH.

Furthermore, using electronic compliance monitors (see supplementary material for details), we correlated time and frequency of pill bottle opening with the number of necessary blood draws to assess whether patients who had difficulty reaching their target FK506 levels (i.e. those requiring more blood draws) had poorer medication compliance (supplementary figure E3). We did not capture the exact time of the blood draw, yet subjects were asked to have the blood checked $24 \mathrm{~h}$ after their last study drug dose to measure FK506 trough levels. There did not appear to be a relationship between compliance and the number of blood draws (Spearman correlation 0.08). Except for one patient in the low-level FK506 arm, all patients had excellent compliance rates of $>85 \%$. However, the data suggest that fewer trough levels were required to reach low and medium FK506 blood levels compared with high levels.

\section{Safety and tolerability of low-dose FK506}

We found that all doses of FK506 were well tolerated (table 2). The most frequent side-effect was nausea/ diarrhoea $(n=11)$, and was observed predominantly in the medium- and high-level FK506 treatment arms. The complaint of diarrhoea was more common in those patients who were on intravenous prostacyclin, although this only led to discontinuation of study medication in one patient. Upper respiratory tract infections and sinus congestion appeared to be more common in the treatment group versus placebo, yet when combined with sinus congestion and cough, they appear to be evenly distributed between the placebo and treatment arms. Of note, we did not observe any line infections in patients who were on i.v. prostacyclins. A total of four events of fluid retention/oedema were noted in four patients. These were mild to moderate in intensity and transient in nature, and did not require initiation or increase in the diuretic regimen. Of the listed adverse events described by intensity by patient, $64 \%$ were judged to be mild 


\begin{tabular}{|c|c|c|c|c|c|}
\hline Parameters & Total cohort & $\begin{array}{l}\text { Placebo } \\
\text { group }\end{array}$ & $\begin{array}{l}\text { Low-level } \\
\text { FK506 group }\end{array}$ & $\begin{array}{l}\text { Medium-level } \\
\text { FK506 group }\end{array}$ & $\begin{array}{l}\text { High-level } \\
\text { FK506 group }\end{array}$ \\
\hline Subjects & 23 & 6 & 6 & 5 & 6 \\
\hline Age years & $41(34-52)$ & $46(38-53)$ & 35 (24-45) & $39(25-51)$ & 45 (42-48) \\
\hline Male/female & $7 / 16$ & $1 / 5$ & $2 / 4$ & $2 / 3$ & $2: 4$ \\
\hline \multicolumn{6}{|l|}{ PAH aetiology } \\
\hline IPAH & 6 & 2 & 2 & 1 & 1 \\
\hline $\mathrm{HPAH}$ & 4 & 1 & 1 & 1 & 1 \\
\hline $\mathrm{D}+\mathrm{T}-\mathrm{APAH}$ & 5 & 1 & 1 & 1 & 2 \\
\hline CTD-APAH & 5 & 2 & 0 & 1 & 2 \\
\hline CHD-APAH & 3 & 0 & 2 & 1 & 0 \\
\hline \multicolumn{6}{|l|}{ NYHA FC } \\
\hline I & 1 & 0 & 0 & 1 & 0 \\
\hline ॥ & 18 & 5 & 5 & 4 & 4 \\
\hline III & 4 & 1 & 1 & 0 & 2 \\
\hline IV & 0 & 0 & 0 & 0 & 0 \\
\hline 6MWD m & $526(472-567)$ & $507(465-577)$ & $551(449-572)$ & $541(475-579)$ & $512(495-525)$ \\
\hline $\begin{array}{c}\text { NT-proBNP } \\
\mathrm{pg} \cdot \mathrm{mL}^{-1}\end{array}$ & 98 (57-328) & 78 (61-87) & $177(62-1036)$ & 98 (47-137) & $398(219-548)$ \\
\hline \multicolumn{6}{|l|}{$\begin{array}{l}\text { Medication } \\
\text { profile }\end{array}$} \\
\hline Prostacyclin" & 18 & 5 & 5 & 4 & 4 \\
\hline $\begin{array}{l}\text { PDE-5 } \\
\text { inhibitor }\end{array}$ & 18 & 4 & 6 & 3 & 5 \\
\hline ERA $^{\pi}$ & 14 & 4 & 5 & 1 & 4 \\
\hline \multicolumn{6}{|l|}{ Therapy } \\
\hline Mono & 6 & 1 & 1 & 3 & 1 \\
\hline Dual & 7 & 3 & 0 & 1 & 3 \\
\hline Triple & 10 & 2 & 5 & 1 & 2 \\
\hline
\end{tabular}

Data are presented as $\mathrm{n}$ or median (interquartile range). PAH: pulmonary arterial hypertension; IPAH: idiopathic PAH; HPAH: hereditary PAH; D+T-PAH: drugs and toxins-associated PAH; CTD-APAH: connective tissue disease-associated PAH; CHD-APAH: congenital heart disease-associated PAH; NYHA FC: New York Heart Association functional class; 6MWD: 6-min walk distance; NT-proBNP: N-terminal brain natriuretic peptide; PDE-5: phosphodiesterase-5; ERA: endothelin receptor antagonist. \#: includes intravenous, subcutaneous and inhaled; ": the ERA used was ambrisentan as patients with bosentan were excluded and macitentan had not yet been approved.

$(n=35), 31 \%$ moderate $(n=17)$ and $5 \%$ severe $(n=3)$. The adverse event intensities are listed in table 2 . Only one patient developed a serious adverse event (i.e. haemoptysis) during the 2-week follow-up period off the study drug that required a brief hospitalisation and imaging studies to verify the bleeding source. This event occurred due to a severe streptococcal throat infection and was treated with antibiotics, and was most likely not related to the study drug, as a different reason was identified (i.e. streptococcal infection) and as the patient was already off drug for a couple of days when the symptoms started.

We did not observe an increase in creatinine $(p=0.369$; figure $3 a)$, decrease in WBC count $(p=0.235$; figure $3 b)$ or decrease in haemoglobin $(p=0.204$; figure $3 c$ ) after the 16 -week treatment period with any of the FK506 doses; rather, mildly increasing haemoglobin levels $\left(<1 \mathrm{~g} \cdot \mathrm{dL}^{-1}\right)$ with increasing FK506 dosing were noted. We did not measure glycated haemoglobin, but random blood glucose levels did not increase during the study period (data not shown).

We also did not observe any side-effects that are known to be associated with immunosuppressive doses of FK506, such as systemic hypertension, acute kidney injury or posterior reversible encephalopathy syndrome. Additionally, we did not observe cardiovascular events, worsening of PAH or hospitalisations for PAH during the study period. Two patients discontinued the clinical trial secondary to adverse effects. One patient in the medium-level FK506 arm reported tactile fever after the first two doses of the study drug and decided to discontinue as he felt he would not be able to tolerate the study drug for 16 weeks. The second patient in the high-level FK506 arm on i.v. prostacyclins discontinued the study after day 6 due to markedly increased diarrhoea compared with her baseline loose stools. Another patient could not commit to regular study visits after changing her job. No formal evaluation was performed at 16 weeks for 
TABLE 2 Adverse and serious adverse events $\#$

\begin{tabular}{|c|c|c|c|c|c|}
\hline & \multirow{2}{*}{$\begin{array}{c}\text { Total cohort } \\
\text { events/ } \\
\text { patients }\end{array}$} & \multicolumn{4}{|c|}{ Adverse events total (mild/moderate/severe) } \\
\hline & & $\begin{array}{l}\text { Placebo } \\
\text { group }\end{array}$ & $\begin{array}{l}\text { Low-level } \\
\text { FK506 group }\end{array}$ & $\begin{array}{l}\text { Medium-level } \\
\text { FK506 group }\end{array}$ & $\begin{array}{l}\text { High-level } \\
\text { FK506 group }\end{array}$ \\
\hline Nausea/diarrhoea & $11 / 9$ & $1(1 / 0 / 0)$ & $0(0 / 0 / 0)$ & $3(2 / 1 / 0)$ & $7(5 / 2 / 0)$ \\
\hline Sinus congestion & $6 / 5$ & $2(1 / 1 / 0)$ & $4(3 / 1 / 0)$ & $0(0 / 0 / 0)$ & $0(0 / 0 / 0)$ \\
\hline $\begin{array}{l}\text { Upper respiratory tract } \\
\text { infection }\end{array}$ & $6 / 5$ & $0(0 / 0 / 0)$ & $4(0 / 4 / 0)$ & $0(0 / 0 / 0)$ & $2(1 / 1 / 0)$ \\
\hline Fluid retention/oedema & $4 / 4$ & $0(0 / 0 / 0)$ & $1(0 / 1 / 0)$ & $0(0 / 0 / 0)$ & $3(2 / 1 / 0)$ \\
\hline Cough & $3 / 3$ & $2(2 / 0 / 0)$ & $1(0 / 0 / 1)$ & $0(0 / 0 / 0)$ & $0(0 / 0 / 0)$ \\
\hline Headache & $3 / 3$ & $1(1 / 0 / 0)$ & $0(0 / 0 / 0)$ & $0(0 / 0 / 0)$ & $2(1 / 0 / 1)$ \\
\hline Infection & $3 / 2$ & $2(2 / 0 / 0)$ & $0(0 / 0 / 0)$ & $0(0 / 0 / 0)$ & $1(0 / 1 / 0)$ \\
\hline Anxiety & $2 / 2$ & $0(0 / 0 / 0)$ & $1(0 / 1 / 0)$ & $0(0 / 0 / 0)$ & $1(1 / 0 / 0)$ \\
\hline Bronchitis & $2 / 2$ & $0(0 / 0 / 0)$ & $1(1 / 0 / 0)$ & $0(0 / 0 / 0)$ & $1(0 / 0 / 1)$ \\
\hline Drug reaction/hives & $2 / 2$ & $0(0 / 0 / 0)$ & $0(0 / 0 / 0)$ & $0(0 / 0 / 0)$ & $2(1 / 1 / 0)$ \\
\hline Flushing & $2 / 2$ & $0(0 / 0 / 0)$ & $1(0 / 1 / 0)$ & $1(1 / 0 / 0)$ & $0(0 / 0 / 0)$ \\
\hline Hand tremors & $2 / 2$ & $0(0 / 0 / 0)$ & $0(0 / 0 / 0)$ & $1(1 / 0 / 0)$ & $1(1 / 0 / 0)$ \\
\hline Chest pain & $1 / 1$ & $0(0 / 0 / 0)$ & $0(0 / 0 / 0)$ & $0(0 / 0 / 0)$ & $1(1 / 0 / 0)$ \\
\hline Fatigue & $1 / 1$ & $0(0 / 0 / 0)$ & $0(0 / 0 / 0)$ & $1(1 / 0 / 0)$ & $0(0 / 0 / 0)$ \\
\hline Fever (tactile) & $1 / 1$ & $0(0 / 0 / 0)$ & $0(0 / 0 / 0)$ & $1(1 / 0 / 0)$ & $0(0 / 0 / 0)$ \\
\hline Halitosis & $1 / 1$ & $0(0 / 0 / 0)$ & $0(0 / 0 / 0)$ & $1(1 / 0 / 0)$ & $0(0 / 0 / 0)$ \\
\hline Insomnia & $1 / 1$ & $0(0 / 0 / 0)$ & $0(0 / 0 / 0)$ & $1(1 / 0 / 0)$ & $0(0 / 0 / 0)$ \\
\hline Musculoskeletal pain & $1 / 1$ & $0(0 / 0 / 0)$ & $1(1 / 0 / 0)$ & $0(0 / 0 / 0)$ & $0(0 / 0 / 0)$ \\
\hline Shortness of breath & $1 / 1$ & $0(0 / 0 / 0)$ & $0(0 / 0 / 0)$ & $0(0 / 0 / 0)$ & $1(1 / 0 / 0)$ \\
\hline Shingles & $1 / 1$ & $0(0 / 0 / 0)$ & $1(0 / 1 / 0)$ & $0(0 / 0 / 0)$ & $0(0 / 0 / 0)$ \\
\hline Stomach cramps & $1 / 1$ & $0(0 / 0 / 0)$ & $0(0 / 0 / 0)$ & $0(0 / 0 / 0)$ & $1(1 / 0 / 0)$ \\
\hline \multicolumn{6}{|l|}{ Serious adverse events } \\
\hline Haemoptysis & $1 / 1$ & $0(0 / 0 / 0)$ & $1(0 / 1 / 0)$ & $0(0 / 0 / 0)$ & $0(0 / 0 / 0)$ \\
\hline
\end{tabular}

The safety profile of FK506 was assessed qualitatively by reporting the type and absolute number of adverse events in the total cohort and the assigned treatment groups. We report the number of events as well as the number of patients experiencing those adverse events. ${ }^{\#}$ : see table 1 for group demographics.

these patients, but all three have had follow-up in our pulmonary hypertension clinic at Stanford and are doing well.

\section{Analysis of clinical, serological and exploratory end-points}

As expected from a small proof-of-concept study that was primarily designed to assess the safety and tolerability and not efficacy of FK506, we did not observe any statistical difference between the four treatment arms with regard to changes in $6 \mathrm{MWD}$ ( $\mathrm{p}=0.475$; figure $4 \mathrm{a})$, NT-proBNP ( $\mathrm{p}=0.283$; figure $4 \mathrm{~b}$ ), DLCO ( $\mathrm{p}=0.113$; figure $4 \mathrm{c})$, RV-FAC $(\mathrm{p}=0.349$; figure $4 \mathrm{~d})$, TAPSE $(\mathrm{p}=0.925$; figure $4 \mathrm{e})$ or RV-GLS
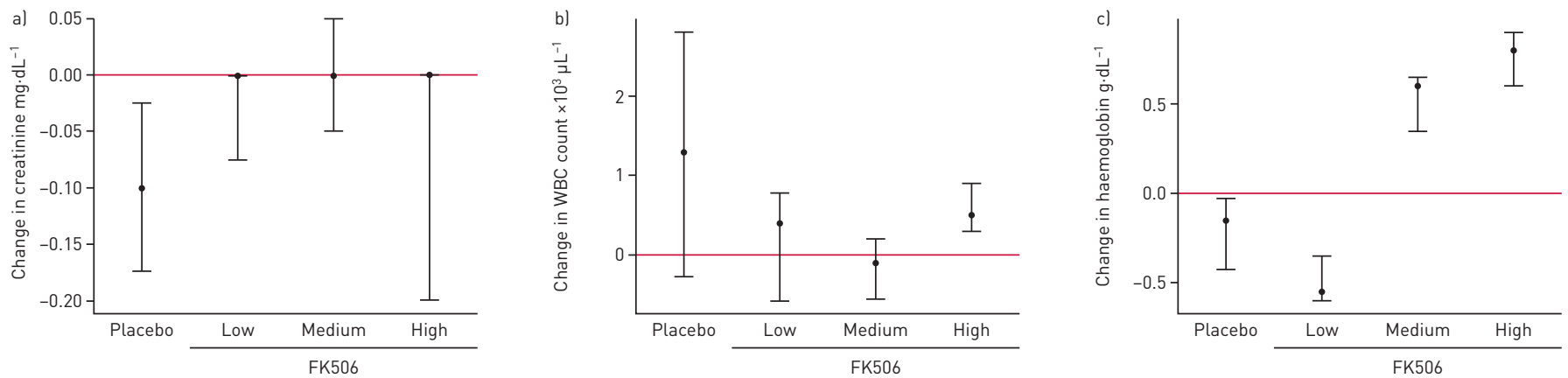

FIGURE 3 Safety parameters: the safety profile of FK506 was assessed quantitatively by measuring the safety parameters a) creatinine, b) white blood cell (WBC) count and c) haemoglobin in the placebo and treatment groups (low, medium and high FK506). The change from baseline is presented as median and interquartile range. The red line represents no change. 

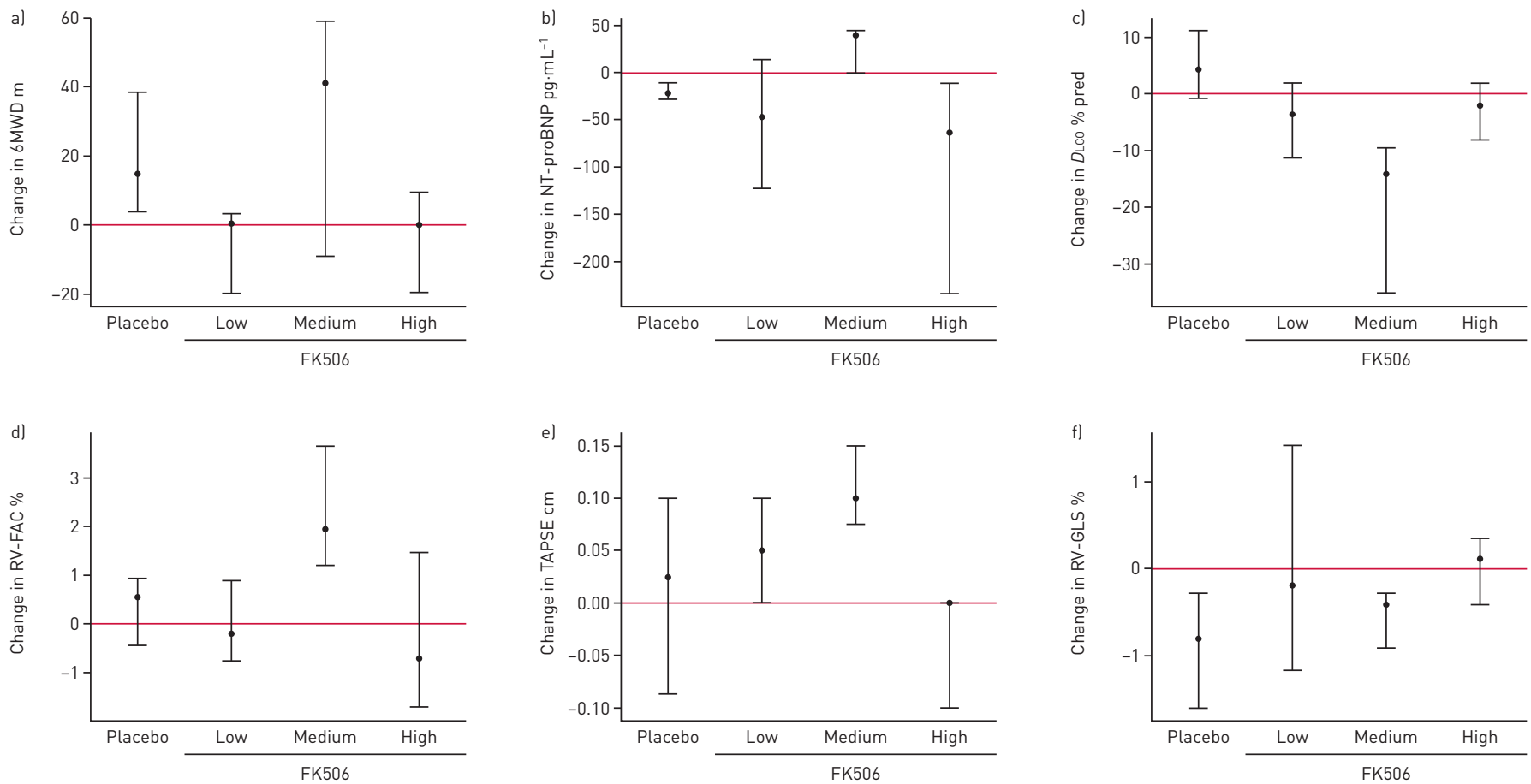

FIGURE 4 Secondary end-points: changes in a) 6-min walk distance (6MWD), b) N-terminal brain natriuretic peptide (NT-proBNP), c) diffusion capacity of the lung for carbon monoxide $(D\llcorner C o), d)$ right ventricular fractional area change (RV-FAC), e) tricuspid annular plane systolic excursion (TAPSE) and f) right ventricular global longitudinal strain (RV-GLS) in the placebo and treatment groups (low, medium and high FK506). Data are presented as median change between baseline and 16 -week follow-up with interquartile range. The red line represents no change.

( $p=0.920$; figure 4f). Similarly, the above secondary end-points were not significantly different in the placebo arm when compared with the combined FK506 treatment groups (low, medium and high) (supplementary tables E1 and E2). As anticipated for a study of small size and short duration in a cohort of stable PAH subjects, we did not observe any TTCW events. Of note, subjects in the medium-level FK506 group appeared to have a different response in terms of the these parameters compared with the other two treatment groups, which we attribute to a larger variability in the medium-level group due to a lower subject number, given that two subjects discontinued in this group.

As exploratory end-points, we looked at serological biomarkers that our research had previously linked to an increase in BMPR2 signalling, including BMPR2, Id1, Cofilin-1, LIMK-1, miR21, miR27a, SMURF-1 and IL-6 (figure 5a) [8]. We did not observe changes in serological biomarkers between the patients who received FK506 and the placebo arm ( $p>0.150$ for all serological biomarkers; supplementary table E2). Although we did not observe statistically significant differences across groups, the observed dose-dependent increase in BMPR2 (figure 5b) as well as an expected decrease in Cofilin-1 (supplementary figure E4a) deserve additional study in the future in a larger sample. We did not observe dose-dependent changes in Id1, LIMK-1, miR21, miR27a, SMURF-1 or IL-6 (figure 5c and supplementary figures E4b-e). When compared with healthy controls, PAH patients at baseline had significantly lower BMPR2 mRNA expression (median (IQR) PAH 0.43 (0.31-0.81) versus controls $0.91(0.78-1.13) ; \mathrm{p}=0.005$ ) (figure 5d) and Id1 expression (median (IQR) PAH 0.24 (0.07-0.43) versus controls $0.69(0.43-0.95) ; \mathrm{p}=0.011)$ (figure 5e) in PBMCs, and BMPR2 and Id1 levels not significantly different from healthy controls after 16 weeks of FK506 treatment $(\mathrm{p}=0.546$ and $\mathrm{p}=0.969$, respectively). Of note, two patients had high BMPR2 expression levels at baseline: one in the placebo and one in the medium-level arm. We cannot exclude an effect of concomitant medication on BMPR2 expression, as we did not control for those before the blood draw.

\section{Subcohort analyses}

In a post hoc analysis, we tried to identify subjects that responded particularly well to FK506 with regard to an increase in BMPR2 expression and looked at their response in some selected clinical parameters: the absolute change in 6MWD, RV-FAC (figure 6a and b), NT-proBNP and RV-GLS (supplementary figure E5a and b). Our choice of echocardiographic parameters was a priori based on our local expertise and publications [14-17]. In a recent study, we validated RV-FAC as a stronger correlate of outcome than TAPSE [14]. As pointed out by the recent study by FINE et al. [18], RV-GLS emerged as the best metric of 

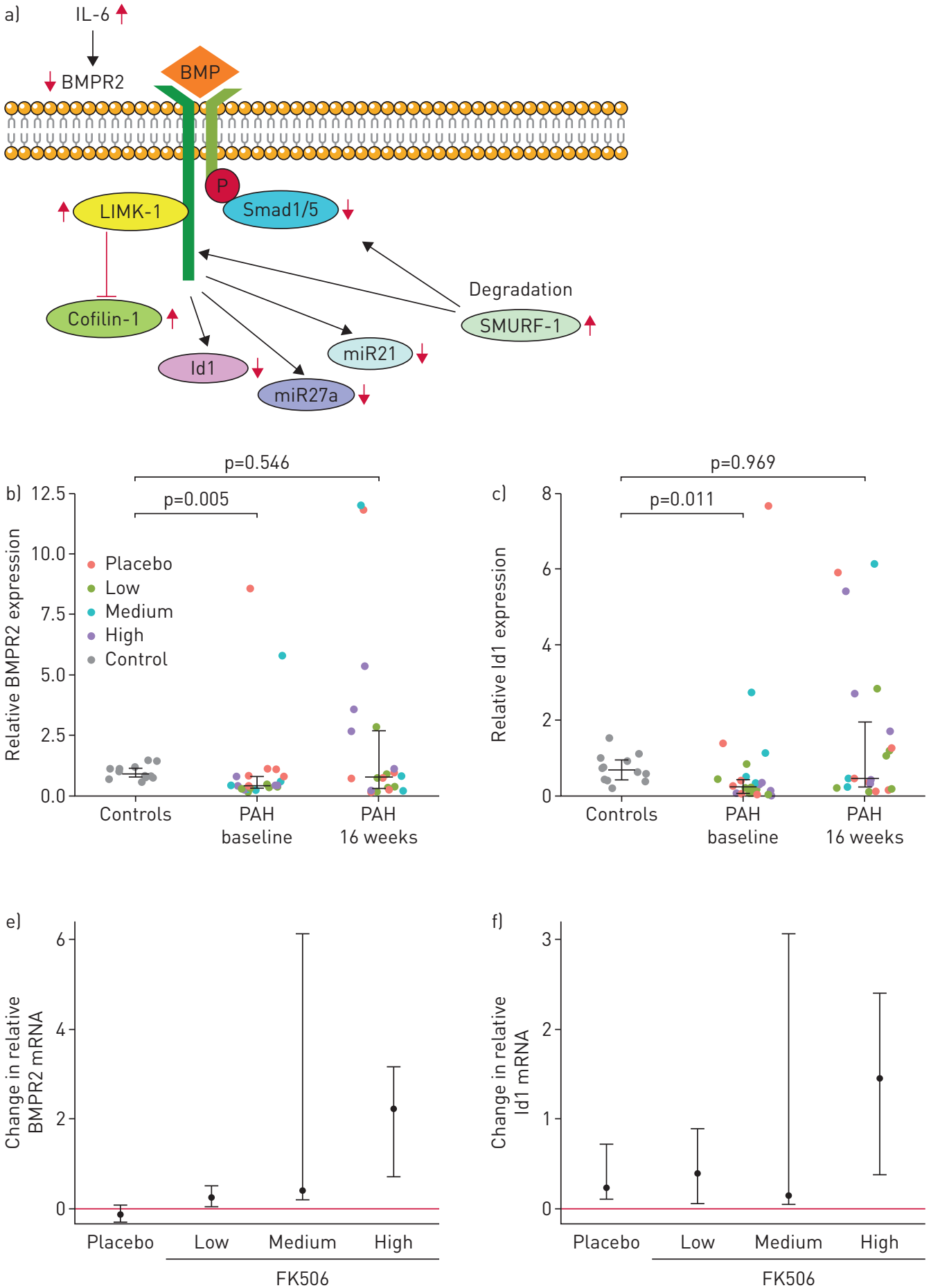

FIGURE 5 Change in bone morphogenetic protein receptor 2 (BMPR2) biomarkers. a) Overview of downstream and upstream targets of BMPR2 known or expected to be altered in pulmonary arterial hypertension (PAH) (red arrows). IL-6: interleukin-6; BMP: bone morphogenetic protein; LIMK-1: Lim domain kinase 1; SMURF-1: Smad ubiquitination regulatory factor 1; Id1: inhibitor of differentiation 1. b, c) RNA expression of b) BMPR2 and c) Id1 in peripheral blood mononuclear cells in healthy controls $(n=13)$ and PAH patients at baseline and 16 weeks after FK506 treatment. RNA expression data presented as relative expression to controls and normalised to glyceraldehyde 3-phosphate dehydrogenase $\left(\Delta \Delta C_{t}\right.$ relative quantification method). Data are presented as median and interquartile range, and compared by the Mann-Whitney-Wilcoxon test. Significance levels are indicated. d, e) Change in d) BMPR2 and e) Id1 expression from baseline to 16-week follow-up in the placebo and treatment groups (low, medium and high FK506). Data are presented as median and interquartile range. The red line represents no change. 

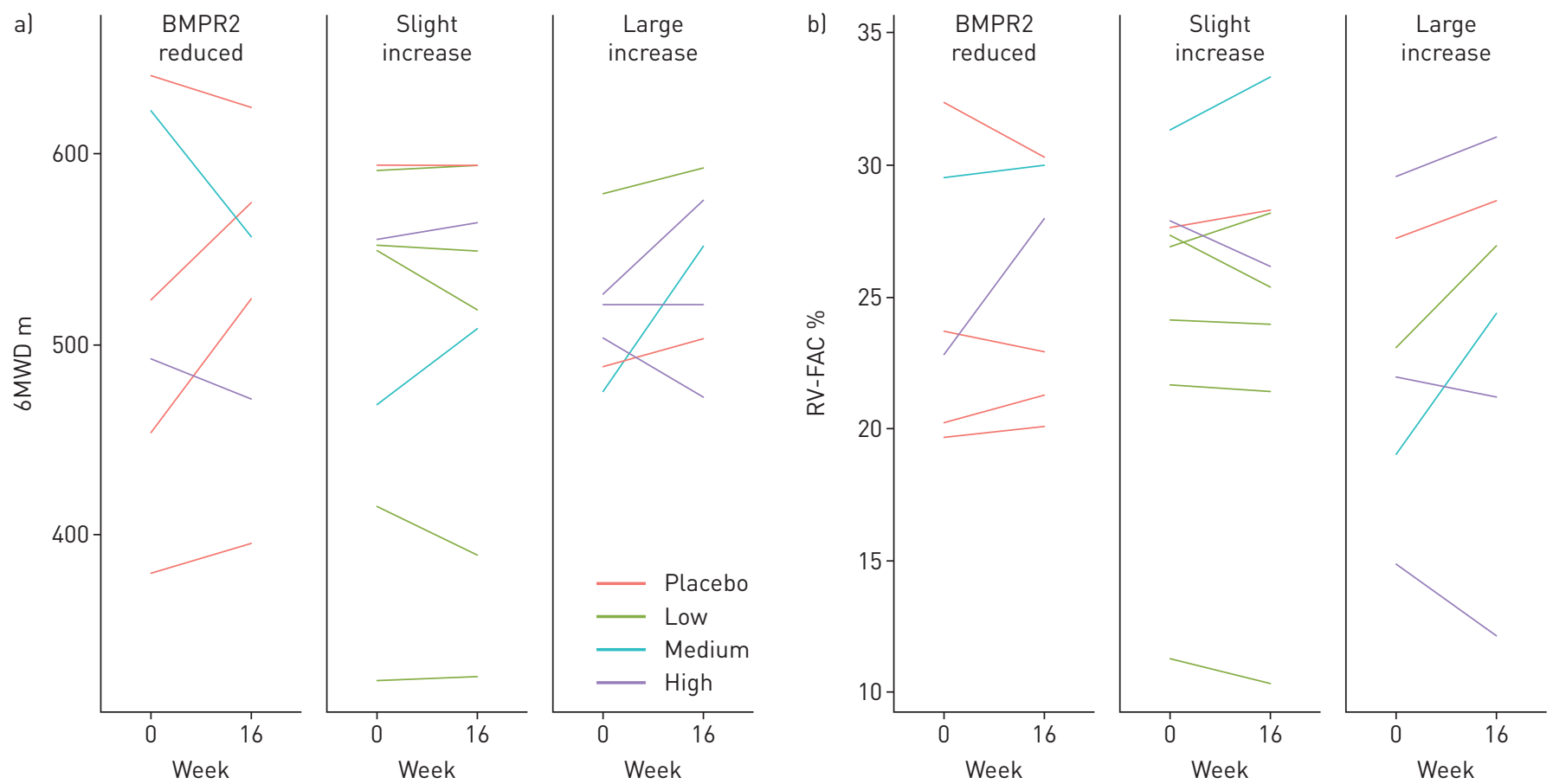

FIGURE 6 Change in bone morphogenetic protein receptor 2 (BMPR2) and clinical response. The line plots show the changes in a) 6-min walk distance (6MWD) and b) right ventricular fractional area change (RV-FAC) in relation to the change in BMPR2 expression. Three groups were defined according to the absolute change in BMPR2 expression in peripheral blood mononuclear cells at 16-week follow-up compared with baseline: BMPR2 unchanged or reduced ("BMPR2 reduced"), a slight increase in BMPR2 (increase in BMPR2 expression but <1, the mean of healthy controls) or a large increase in BMPR2 (increase in BMPR2 expression >1).

right ventricular function when compared with RV-FAC and TAPSE in PAH; ongoing studies are currently validating the findings in independent cohorts. Subjects were grouped in three groups depending on the absolute change in BMPR2: no change or reduction in BMPR2, a slight increase in BMPR2 (increase in BMPR2 expression but $<1$, the mean of healthy controls) or a large increase in BMPR2 (increase in BMPR2 expression >1). While we found an association between a BMPR2 increase and an improvement in the above clinical parameters in some patients, this association was not present in all patients.

Vice versa, we also looked whether some subjects differed from others with respect to their clinical response and whether this difference was at all related to a change in BMPR2 expression. As a potential beneficial clinical effect, we defined a change in $6 \mathrm{MWD}$ of $\geqslant 40 \mathrm{~m}$, and/or a reduction in NT-proBNP of $\geqslant 200 \mathrm{pg} \cdot \mathrm{mL}^{-1}$, and/or an increase in RV-FAC of $\geqslant 1.8 \%$, and/or decrease in RV-GLS of $\geqslant 2.5 \%$. Using the above criteria, 10 out of 17 subjects who completed the clinical trial showed a beneficial clinical effect. There was no statistically significant difference in BMPR2 and Id1 expression $(p=0.565$ and $p=0.250$, respectively; supplementary figure E6a and b) in subjects who showed some clinical benefit compared with the clinical "nonresponders".

We included four patients with HPAH and evaluated the effect of FK506 on BMPR2 expression in HPAH. Based on our pre-clinical work, we hypothesised that FK506 would be effective even in patients with a $B M P R 2$ mutation [2]. We had shown that in the case of a BMPR2 mutation, a different type 2 receptor, i.e. the activin receptor type II A, was recruited to the type 1 receptors and downstream signalling was restored [2]. Only the one BMPR2 mutant patient receiving high-level FK506 had an increase in BMPR2 expression, whereas the two HPAH patients randomised to the low- and medium-level FK506 arms had no change in BMPR2 expression (supplementary figure E7). A possible explanation for the different response to FK506 in the three HPAH patients could be different BMPR2 mutations or the presence of other modifier genes such as FKBP12 (FK506 binding protein), which interfere with the mode of action of FK506 [2]. Further study would be required to test FK506 in a larger number of patients to conclusively evaluate its effect in HPAH.

\section{Discussion}

This is the first report of a phase IIa randomised, safety and tolerability trial using low-level FK506 in PAH. We show that it is feasible to target and maintain low FK506 levels in PAH patients $\left(<5 \mathrm{ng} \cdot \mathrm{mL}^{-1}\right)$ and that those levels are well tolerated. Our study was not powered to demonstrate efficacy of FK506, and 
we did not observe changes in serological biomarkers between the patients who received FK506 and the placebo arm. Although not the main aim of this study, we demonstrate that PAH patients at baseline express significantly lower BMPR2 and Id1 in PBMCs compared with healthy controls.

While our pre-clinical studies documented FK506 blood levels of $0.2 \mathrm{ng} \cdot \mathrm{mL}^{-1}$ to be effective in reducing experimental pulmonary hypertension [2], we chose $2 \mathrm{ng} \cdot \mathrm{mL}^{-1}$ as our lowest FK506 target level as this was the lowest detection limit of clinically approved FK506 assays. Given the good correlation between FK506 doses and blood levels, it might be feasible to use fixed low FK506 doses instead of targeting certain FK506 blood levels in future studies. We show that low-level FK506 $\left(<5 \mathrm{ng} \cdot \mathrm{mL}^{-1}\right)$ has the advantage of not inducing commonly observed serious systemic side-effects related to immunosuppressive doses of FK506, such as nephrotoxicity, systemic hypertension, diabetes, anaemia or infections, a finding that might be confounded by the relatively short observation period of just 16 weeks. Of note, the most common side-effect, i.e. nausea/diarrhoea, was predominantly observed in our highest FK506 arm (3-5 ng. $\left.\mathrm{mL}^{-1}\right)$, emphasising the need to identify the lowest effective dose of FK506 for PAH in future studies. Of importance, we specifically did not observe any central line infections in patients on concomitant i.v. prostacyclin therapy. Furthermore, there is evidence that low-level FK506 might have additional distinct, yet understudied, properties such as inducing T-regulatory cells [19], which have been shown to be beneficial in experimental pulmonary hypertension [20, 21], skewing bone marrow-derived macrophage polarisation towards a M2 macrophage phenotype [22], as well as inducing apoptosis in vascular smooth muscle cells [2] and blocking atherosclerosis in $\mathrm{ApoE}^{-/-}$mice [23].

Although it is known that phosphodiesterase-5 (PDE-5) inhibitors and tacrolimus compete for metabolism at CYP3A4, we did not see any evidence of increased PDE- 5 concentrations as manifested by increased side-effects such as hypotension, headaches or flushing. We did, however, observe that subjects on high-dose PDE-5 inhibitors (>60 mg three times daily) only required minimal doses of FK506 to achieve their goal blood levels, suggesting some interaction between the two drugs. We excluded patients on bosentan therapy for safety reasons for this study, as the bosentan drug label described a potential interaction between tacrolimus and bosentan resulting in higher bosentan plasma levels in animals. Yet, as these findings have not been confirmed in humans, a future, well-monitored FK506 clinical trial might very well include patients on bosentan.

Our study has several limitations. It was performed at a single centre, our sample size was small and we included a more heterogeneous group of subjects than we would have liked: stable NYHA functional class II as well as NYHA functional class III PAH patients on multiple PAH treatment regimen. In order to determine whether FK506 might be efficacious in PAH, we believe that a larger, more homogeneous sample size, potentially including subjects with more advanced disease and longer treatment and observation time, would be required, i.e. subjects similar to the three end-stage PAH patients (NYHA functional class III/IV) who received compassionate use of FK506 and who were followed for over 1 year [8]. A further limitation of our study is that we provide incomplete follow-up of the three patients who discontinued the study.

One of the goals of this study was to identify blood biomarkers that would reflect BMPR2 deficiency as well as response to FK506 treatment to guide us in enrichment strategies for a future phase IIb/III efficacy trial. PAH patients as a group had $\sim 50 \%$ lower BMPR2 expression in PBMCs compared with healthy controls, which increased after FK506 treatment. PBMCs have been used in the past as surrogates for PAH and lung pathobiology [24]. Hemnes et al. [24] recently reported that the gene expression signature derived from cultured lymphocytes was able to discriminate two clinically and potentially histopathologically different forms of $\mathrm{PAH}$, i.e. vasoreactive (also termed reversible) and nonvasoreactive (fixed) $\mathrm{PAH}$. Differentially expressed genes in lymphocytes included genes important in PAH lung pathobiology such as cytoskeleton and cell-cell adhesion factors, suggesting that the gene signature in circulating immune cells might indeed reflect lung pathology. To date, however, it is not known whether reduced BMPR2 expression in PBMCs reflects the BMPR2 deficiency observed in multiple organs in PAH and how BMPR2 is regulated. Ideally, future studies should identify a surrogate biomarker of BMPR2 deficiency (in expression or signalling) with the goal to define a target patient population that might benefit from a BMPR2-modulating therapy such as FK506.

Furthermore, BMPR2 expression in PBMCs might not be the best surrogate biomarker to assess the effect of FK506, given that our previous data had predominantly shown that FK506 mechanistically increases BMPR2 signalling rather than expression [2]. A future trial therefore potentially requires the inclusion of other biomarkers such as Smad phosphorylation to assess response to therapy.

While we observed that some subjects responded with a more pronounced increase in BMPR2 expression than others, which was associated with an improvement in clinical end-points such as 6MWD, 
NT-proBNP, RV-FAC and RV-GLS in some subjects, those changes were not significant, limiting the overall interpretation.

In conclusion, low-level FK506 is safe and increases BMPR2 expression in subsets of PAH patients. These results are encouraging to further evaluate the role of FK506 in BMPR2-deficient PAH patients in a larger, multicentre trial.

\section{Acknowledgements}

Data Safety and Monitoring Board members: Steven M. Kawut (Perelman School of Medicine, University of Pennsylvania, Philadelphia, PA, USA), Stephen C. Mathai (Johns Hopkins University School of Medicine, Baltimore, MD, USA) and Benjamin A. Goldstein (Stanford University School of Medicine, Stanford, CA, USA).

Author contributions: Substantial contributions to the conception or design of the work: E. Spiekerkoetter, R.T. Zamanian, J. Long-Boyle; acquisition of data: Y.K. Sung, D. Sudheendra, V. Scott, P. Del Rosario, M. Bill, E. Spiekerkoetter, R.T. Zamanian; analysis or interpretation of data: H. Hedlin, F. Haddad, E. Spiekerkoetter, R.T. Zamanian; drafting the work: E. Spiekerkoetter, R.T. Zamanian; or revising it critically for important intellectual content: E. Spiekerkoetter, R.T. Zamanian, Y.K. Sung. Final approval of the version to be published: all authors

\section{References}

1 Farber HW, Miller DP, Poms AD, et al. Five-year outcomes of patients enrolled in the reveal registry. Chest 2015; 148: 1043-1054.

2 Spiekerkoetter E, Tian X, Cai J, et al. FK506 activates BMPR-II, rescues endothelial dysfunction, and reverses pulmonary hypertension. J Clin Invest 2013; 123: 3600-3613.

3 Long L, Ormiston ML, Yang X, et al. Selective enhancement of endothelial BMPR-II with BMP9 reverses pulmonary arterial hypertension. Nat Med 2015; 21: 777-785.

4 Atkinson C, Stewart S, Upton PD, et al. Primary pulmonary hypertension is associated with reduced pulmonary vascular expression of type II bone morphogenetic protein receptor. Circulation 2002; 105: 1672-1678.

5 Thomson JR, Machado RD, Pauciulo MW, et al. Sporadic primary pulmonary hypertension is associated with germline mutations of the gene encoding BMPR-II, a receptor member of the TGF-beta family. J Med Genet 2000; 37: 741-745.

6 International PPH Consortium, Lane KB, Machado RD, et al. Heterozygous germline mutations in BMPR-II, encoding a TGF-beta receptor, cause familial primary pulmonary hypertension. Nat Genet 2000; 26: 81-84.

7 Deng Z, Morse JH, Slager SL, et al. Familial primary pulmonary hypertension (gene PPH1) is caused by mutations in the bone morphogenetic protein receptor-II gene. Am J Hum Genet 2000; 67: 737-744.

8 Spiekerkoetter E, Sung YK, Sudheendra D, et al. Low-dose FK506 (tacrolimus) in end-stage pulmonary arterial hypertension. Am J Respir Crit Care Med 2015; 192: 254-257.

9 Reynolds AM, Holmes MD, Danilov SM, et al. Targeted gene delivery of BMPR-II attenuates pulmonary hypertension. Eur Respir J 2012; 39: 329-343.

10 Reynolds AM, Xia W, Holmes MD, et al. Bone morphogenetic protein type 2 receptor gene therapy attenuates hypoxic pulmonary hypertension. Am J Physiol Lung Cell Mol Physiol 2007; 292: L1182-L1192.

11 Mathai SC, Puhan MA, Lam D, et al. The minimal important difference in the 6-minute walk test for patients with pulmonary arterial hypertension. Am J Respir Crit Care Med 2012; 186: 428-433.

12 Gruber SA, Hewitt JM, Sorenson AL, et al. Pharmacokinetics of FK506 after intravenous and oral administration in patients awaiting renal transplantation. J Clin Pharmacol 1994; 34: 859-864.

13 Venkataramanan R, Swaminathan A, Prasad T, et al. Clinical pharmacokinetics of tacrolimus. Clin Pharmacokinet 1995; 29: 404-430.

14 Shiran H, Zamanian RT, McConnell MV, et al. Relationship between echocardiographic and magnetic resonance derived measures of right ventricular size and function in patients with pulmonary hypertension. J Am Soc Echocardiogr 2014; 27: 405-412.

15 Vonk-Noordegraaf A, Haddad F, Chin KM, et al. Right heart adaptation to pulmonary arterial hypertension: physiology and pathobiology. J Am Coll Cardiol 2013; 62: D22-D33.

16 Forfia PR, Fisher MR, Mathai SC, et al. Tricuspid annular displacement predicts survival in pulmonary hypertension. Am J Respir Crit Care Med 2006; 174: 1034-1041.

17 Haddad F, Spruijt OA, Denault AY, et al. Right heart score for predicting outcome in idiopathic, familial, or drugand toxin-associated pulmonary arterial hypertension. JACC Cardiovasc Imaging 2015; 8: 627-638.

18 Fine NM, Chen L, Bastiansen PM, et al. Outcome prediction by quantitative right ventricular function assessment in 575 subjects evaluated for pulmonary hypertension. Circ Cardiovasc Imaging 2013; 6: 711-721.

19 Wang $\mathrm{Z}$, Shi B, Jin $\mathrm{H}$, et al. Low-dose of tacrolimus favors the induction of functional $\mathrm{CD} 4^{+} \mathrm{CD} 25^{+} \mathrm{FoxP} 3^{+}$ regulatory T cells in solid-organ transplantation. Int Immunopharmacol 2009; 9: 564-569.

20 Tamosiuniene R, Nicolls MR. Regulatory T cells and pulmonary hypertension. Trends Cardiovasc Med 2011; 21: 166-171.

21 Tamosiuniene R, Tian W, Dhillon G, et al. Regulatory $\mathrm{T}$ cells limit vascular endothelial injury and prevent pulmonary hypertension. Circ Res 2011; 109: 867-879.

22 Bai L, Gabriels K, Wijnands E, et al. Low- but not high-dose FK506 treatment confers atheroprotection due to alternative macrophage activation and unaffected cholesterol levels. Thromb Haemost 2010; 104: 143-150.

23 Donners MM, Bot I, De Windt LJ, et al. Low-dose FK506 blocks collar-induced atherosclerotic plaque development and stabilizes plaques in ApoE ${ }^{-1-}$ mice. Am J Transplant 2005; 5: 1204-1215.

24 Hemnes AR, Trammell AW, Archer SL, et al. Peripheral blood signature of vasodilator-responsive pulmonary arterial hypertension. Circulation 2015; 131: 401-409. 